\title{
Yelp for Prescribers: A Quasi-Experimental Study of Providing Antibiotic Cost Data and Prescription of High-Cost Antibiotics in an Academic and Tertiary Care Hospital
}

\author{
Kira L. Newman, B.A. 1,2 , Jay Varkey, M.D., M.P.H., ${ }^{2,3}$, Justin Rykowski, B.S. ${ }^{2,4}$, and Arun V. Mohan, M.D., \\ M.B.A. ${ }^{2,4,5}$ \\ 'Department of Epidemiology, Emory University Rollins School of Public Health, Atlanta, GA, USA; ${ }^{2}$ Emory University School of Medicine, Atlanta, GA, \\ USA; ${ }^{3}$ Division of Infectious Diseases, Department of Medicine, Emory University School of Medicine, Atlanta, GA, USA; ${ }^{4}$ Division of Hospital \\ Medicine, Department of Medicine, Emory University School of Medicine, Atlanta, GA, USA; ${ }^{5}$ ApolloMD, Atlanta, GA, USA.
}

BACKGROUND: Physicians frequently prescribe antibiotics to inpatients without knowledge of medication cost. It is not well understood whether providing cost data would change prescribing behavior.

OBJECTIVE: To evaluate the association between providing cost data alongside culture and antibiotic susceptibility results and prescribing of high-cost antibiotics.

DESIGN: Quasi-experimental pre-post analysis.

PARTICIPANTS: Inpatients diagnosed with bacteremia or urinary tract infection in two tertiary care hospitals.

INTERVENTION: Cost category data for each antibiotic (\$, \$\$, $\mathbf{\$} \$$, or $\mathbf{\$} \$ \mathbf{S}$ ) were added to culture and susceptibility testing results available to physicians.

MAIN MEASURES: Average cost category of antibiotics prescribed to patients after the receipt of susceptibility testing results.

KEY RESULTS: There was a significant decrease in the average cost category of antibiotics per patient after the intervention (pre-intervention $=1.9 \$ \mathrm{vs}$. postintervention $=1.7 \$$, where $1.5 \$$ would mean that the average number of dollar signs for antibiotics prescribed was between $\$$ and $\$ \$, p=0.002)$. After adjusting for age, insurance type, and prior length of stay, the odds ratio (OR) of a patient's average antibiotic being higher cost vs. lower cost after the intervention compared to before the intervention was 0.74 [95 \% confidence interval (CI) 0.56 , 0.98]. The intervention was associated with a $31.3 \%$ reduction in the average cost per unit of antibiotics prescribed $(p<0.001)$.

CONCLUSIONS: Providing physicians with cost feedback alongside susceptibility testing data was associated with a significant decrease in prescription of high-cost antibiotics. This intervention is intuitive, low cost, and may shift providers toward lower cost medications when equally acceptable options are available.

KEY WORDS: infectious disease; hospital medicine; health care costs, medical decision making.

Electronic supplementary material The online version of this article (doi:10.1007/s11606-015-3253-2) contains supplementary material, which is available to authorized users.

Received July 28, 2014

Revised January 14, 2015

Accepted February 9, 2015

Published online March 7, 2015
J Gen Intern Med 30(8):1140-6

DOI: $10.1007 / \mathrm{s} 11606-015-3253-2$

(c) Society of General Internal Medicine 2015

\section{INTRODUCTION}

Health care costs in the USA have been growing at an unsustainable rate. ${ }^{1}$ As a result, there has been interest in strategies to reduce health care costs while preserving or improving patient outcomes. One approach supported by federal health policy is the use of electronic health records (EHRs). ${ }^{2}$ Proponents argue that EHRs would lead to better quality at lower cost by reducing inefficiencies, improving care coordination and reducing medical errors. ${ }^{3,4}$

Despite enthusiasm for EHRs, evidence of their efficacy at reducing health care costs is mixed. ${ }^{5-9}$ These data indicate that simply implementing an EHR system is not sufficient to control costs. ${ }^{10}$ Instead, EHRs may need to incorporate clinical decision support to guide providers toward cost-saving choices.

Previous interventions to reduce costs associated with diagnostic tests by providing data related to the cost of the test through the EHR have been successful. ${ }^{5,11,12}$ However, results from early attempts to encourage physicians to provide lower cost medications through decision support tools have had mixed success. A study by Ornstein et al. ${ }^{13}$ reported that providing medication cost information in the EHR of a family-practice clinic did not change the overall cost of medications prescribed. Decreased expenditures on certain classes of medications counterbalanced an increased mean cost of other agents. However, a study that examined the effect of providing a computerized support tool to encourage appropriate antibiotic therapy for inpatients found that it reduced the hospital's antimicrobial costs, ${ }^{14}$ and a recent study of community-based practices in Massachusetts showed that a formulary decision support tool was associated with significant cost savings overall. ${ }^{15}$ Real-time feedback about cost data may be more effective in specific settings and for certain drug categories. 
On May 26, 2010, we added a feature to the EHR to make providers aware of antibiotic costs. The purpose of our analysis was to determine whether presenting cost data at the time of antibiotic ordering was associated with a decrease in utilization of high-cost antibiotics.

\section{METHODS}

\section{Study Design and Setting}

This study was a retrospective quasi-experimental pre-post analysis conducted using records from Emory University Hospital and Emory University Hospital Midtown, both urban academic hospitals in Atlanta, Georgia. We collected records of admitted patients diagnosed with bacteremia or urinary tract infection (UTI) between May 26, 2009, through May 25, 2011-12 months before and 12 months after the deployment of the computerized decision support tool. The Institutional Review Board of Emory University approved the study (IRB00061277).

\section{Intervention}

Since 2007, Emory University hospitals have used an EHR and computerized order entry system (PowerChart, Cerner Corporation). The system reports a table of antibiotic susceptibility data alongside microbial culture results for all positive cultures. Beginning May 26, 2010, cost category data for antibiotics were added to the table. When physicians received microbial culture results, the table of antibiotic susceptibilities included a cost category ranking from $\$$ to $\$ \$ \$$ for each drug (Fig. 1, Supplementary Table 1). The number of symbols for each antibiotic was assigned based on cut points determined by the per unit cost of each antibiotic to the hospital at that time. The decision to use cost categories rather than actual cost was based on ease of implementation, simplicity, evidence from the social sciences literature suggesting that cost scales may facilitate decision-making, ${ }^{16}$ feedback from providers, and the fact that while antibiotic cost may change frequently, cost categories tend to be static. To implement the intervention, a cost category indicator was appended to the antibiotic name.

The pre-intervention period began May 26, 2009, and lasted 12 months. It was followed by the implementation of the cost feature for all EHRs. The post-intervention observation period began May 26, 2010, and lasted 12 months. During this period, no changes were made to the cost categories.
Table 1 Comparison of Characteristics of the Pre- and PostIntervention Groups

\begin{tabular}{|c|c|c|c|}
\hline \multirow[t]{2}{*}{ Characteristic } & $\begin{array}{l}\text { Pre- } \\
\text { Intervention } \\
(n=341)\end{array}$ & $\begin{array}{l}\text { Post- } \\
\text { intervention } \\
(n=311)\end{array}$ & \multirow[t]{2}{*}{$p$-value } \\
\hline & No. (\%) & No. $(\%)$ & \\
\hline Age $($ mean $\pm \mathrm{SD})$ & $58.3(17.4)$ & $58.7(16.7)$ & 0.724 \\
\hline Male & $158(46.3)$ & $173(49.2)$ & 0.018 \\
\hline Race & - & - & 0.347 \\
\hline White & $172(50.4)$ & $168(47.7)$ & - \\
\hline Black & $141(41.4)$ & $112(31.8)$ & - \\
\hline Other & $28(8.2)$ & $31(8.8)$ & - \\
\hline Insurance type & - & - & 0.350 \\
\hline Public & $214(62.8)$ & $209(67.2)$ & - \\
\hline Private & $110(32.3)$ & $92(29.6)$ & - \\
\hline Other & $17(5.0)$ & $10(3.2)$ & - \\
\hline $\begin{array}{l}\text { Time until susceptibility } \\
\text { results from date of } \\
\text { admission, days } \\
(\text { mean } \pm \text { SD) }\end{array}$ & $8.7(10.4)$ & $7.8(10.6)$ & 0.779 \\
\hline $\begin{array}{l}\text { Total length of stay, days } \\
(\text { mean } \pm \text { SD) }\end{array}$ & $16.3(15.1)$ & $15.8(17.2)$ & 0.669 \\
\hline $\begin{array}{l}\text { SOI on admission } \\
(\text { mean } \pm \text { SD })\end{array}$ & $3.2(0.7)$ & $3.2(0.9)$ & 0.037 \\
\hline $\begin{array}{l}\text { ROM on admission } \\
(\text { mean } \pm \mathrm{SD})\end{array}$ & $2.6(0.9)$ & $2.7(1.0)$ & 0.211 \\
\hline \multicolumn{4}{|l|}{$\begin{array}{l}\text { (mean } \pm \text { SD) } \\
\text { Non-antibiotic medications }\end{array}$} \\
\hline Anticoagulants ${ }^{\dagger}$ & $56(16.4)$ & $42(13.5)$ & 0.298 \\
\hline Beta blockers & $82(24.6)$ & $59(19.0)$ & 0.116 \\
\hline Calcium channel & $44(12.9)$ & $30(9.7)$ & 0.190 \\
\hline \multicolumn{4}{|l|}{ blockers } \\
\hline Diabetes medications & $68(19.9)$ & $66(21.2)$ & 0.686 \\
\hline Diuretics ${ }^{\S}$ & $20(5.9)$ & $16(5.1)$ & 0.688 \\
\hline $\begin{array}{c}\text { Other anti- } \\
\text { hypertensives }\end{array}$ & $16(4.7)$ & $19(6.1)$ & 0.423 \\
\hline UTI diagnosis & 83 & 72 & 0.823 \\
\hline $\begin{array}{l}\text { Antibiotics prescribed per } \\
\text { person after susceptibility } \\
\text { testing }\end{array}$ & - & - & - \\
\hline 1 & $261(76.5)$ & $246(79.1)$ & 0.364 \\
\hline 2 & $62(18.2)$ & $55(17.7)$ & - \\
\hline 3 & $14(4.1)$ & $7(2.3)$ & - \\
\hline 4 & $4(1.2)$ & $2(0.6)$ & - \\
\hline 5 & $0(0.0)$ & $1(0.3)$ & - \\
\hline $\begin{array}{l}\text { Mean cost per person, } \$ \\
(\text { mean } \pm \text { SD) }\end{array}$ & $1.9(0.7)$ & $1.7(0.6)$ & 0.004 \\
\hline
\end{tabular}

SD standard deviation, SOI severity of illness score, ROM risk of mortality score, UTI urinary tract infection

*Number of individuals prescribed each class or category of medication ${ }^{\prime}$ Includes warfarin, heparin, factor Xa inhibitors, and direct thrombin inhibitors

${ }^{7}$ Includes insulin, sulfonylureas, meglitinides, metformin, and DPP-4 inhibitors $\S$ Includes thiazides, loop diuretics, potassium-sparing diuretics, and carbonic anhydride inhibitors

"Includes clonidine, select vasodilators, angiotensin converting enzyme inhibitors, and angiotensin II receptor blockers

"Includes 84 individuals diagnosed with both bacteremia and urinary tract infection

\section{Participants}

Patients were eligible for the study if they had been diagnosed with bacteremia (ICD-9 codes: 038.x, 022.3, 790.7, 054.5,

\begin{tabular}{|lcc|}
\hline & & Enterococcus Faecalis \\
\hline Drug & Interpretation \\
\hline Ampicillin $\$$ & SUSCEPTIBLE \\
\hline Nitrofurantoin & $\$$ & SUSCEPTIBLE \\
\hline Penicillin $\$$ & SUSCEPTIBLE \\
\hline Tetracycline & $\$$ & Resistant \\
\hline Vancomycin & $\$ \$$ & SUSCEPTIBLE \\
\hline
\end{tabular}

Fig. 1 Example of a cost feedback screen provided in susceptibility testing results 
036.2, 003.1, and 020.2) or UTI (ICD-9 codes: 599.x, 590.x, 595.x, and 597.x) during a hospitalization, had received susceptibility testing to confirm their diagnosis, had positive culture results that indicated susceptibility to at least two cost classes of antibiotics (e.g., \$ and \$\$), and had been prescribed antibiotics following susceptibility testing results. These conditions were selected because of their incidence and the variety of prescribing options available. For patients with multiple hospital stays during the period, we only included the first visit in our analysis because patients may have been more likely to receive the same drug regimen during subsequent hospital stays. For patients with both bacteremia and UTI diagnoses, we considered prescriptions after the second positive culture result to be eligible for inclusion, regardless of sample type, to minimize inclusion of antibiotics prescribed for prior infections. During diagnosis-stratified analyses, we included dual-diagnosis individuals in both groups, but we only included prescriptions from after the culture result specific to the stratum (i.e., urine for UTI, blood for bacteremia).

\section{Outcome Measures and Data Collection}

The primary outcome was the average cost of antibiotics prescribed after susceptibility testing, as measured in the number of $\$$ symbols. Additional outcomes included the number of antibiotics prescribed and the prescription of high-cost versus lowcost antibiotics (\$ and \$ vs. \$\$ and \$ \$ \$). For all outcomes, we compared data before and following the intervention.

Sources of data for this study included administrative and clinical data from the EHR and discharge data from the University Health System Consortium (UHC). Administrative and clinical records were used to collect demographic, medication, and laboratory data. UHC provided severity of illness (SOI) and risk of mortality (ROM) data, as determined by the All Patient Refined Diagnosis Group (APR-DRG) Grouper. ${ }^{17}$

\section{Statistical Analysis}

We used paired t-tests for continuous variables, $\chi^{2}$ tests for categorical variables, and $\chi^{2}$ tests for trends for ordinal variables. Our primary analysis was a multivariable logistic regression model to estimate the association between high-cost antibiotic use (\$\$ and $\$ \$ \$ \$$ ) and the cost-feedback intervention (pre vs. post), with low average cost of antibiotic prescribing (\$ and \$ $\$$ ) as the reference group. We adjusted for other possible exposures influencing prescribing, including sex, race, age, insurance status, length of hospital stay prior to antibiotic prescribing, creatinine, SOI, and ROM. Using the estimated odds ratio (OR) from that model as the standard, we removed exposure variables that increased the precision of our estimate, as measured by the ratio of the $95 \%$ confidence interval (CI), but did not change the OR by more than $10 \%$ from the standard. ${ }^{18} \mathrm{We}$ evaluated for goodness of fit using the Hosmer-Lemeshow test. We also compared the average cost per unit of antibiotics prescribed pre- and post-intervention as estimated using the median antibiotic cost per cost category.
SAS 9.3 (SAS Institute, Cary, NC) was used for all analyses. $P$-values $<0.05$ were considered statistically significant.

\section{Sensitivity Analyses}

To assess our study results' robustness, we conducted a sensitivity analysis. To evaluate the impact of our primary metric (average cost of antibiotics prescribed), we repeated our analyses using the highest cost and then the lowest cost postsensitivity testing antibiotic prescribed for each individual. Second, we stratified our data by diagnosis (bacteremia or UTI) and repeated our analyses.

\section{RESULTS}

\section{Cost Categorization}

The antibiotics were each assigned to one of four cost categories. The median cost per unit for $\$$ category antibiotics was \$2.64 (IQR: \$0.81-\$5.78). For \$ category antibiotics, the median cost per unit was \$7.66 (IQR: \$6.09-\$9.19). For \$\$ category antibiotics, the median cost per unit was $\$ 15.19$ (IQR: $\$ 12.10-\$ 16.85)$. For $\$ \$ \$ \$$ category antibiotics, the median cost per unit was $\$ 87.43$ (IQR: $\$ 67.48-\$ 147.02$ ).

\section{Participant Characteristics}

Of 1,471 individuals with diagnoses of bacteremia and prescriptions for antibiotics, 581 people had a positive test result susceptible to antibiotics from more than one cost category and were prescribed antibiotics following that test, making them eligible for inclusion (Fig. 2). Of 3,902 individuals with diagnoses of UTI or related conditions and prescriptions for antibiotics, 155 people were diagnosed with a UTI. Of the 581 eligible individuals with bacteremia, 84 were also diagnosed with a UTI (Table 1). Of those diagnosed with both, 67 people were diagnosed with bacteremia first and 17 were diagnosed with a UTI first. The characteristics of participants in the preand post-intervention periods were similar, although there were significant differences in the proportion of males and SOI on admission. Overall, their mean age was 58.5 years, $50.7 \%$ were male, and $52.2 \%$ were white. Most had public insurance $(64.9 \%)$. Most had severe illnesses upon admission (overall mean $\mathrm{SOI}=3.2$ ), and their average length of stay was long (overall mean=16.1 days), although individual durations varied greatly (overall standard deviation $(\mathrm{SD})=16.1$ days).

\section{Antibiotic Prescribing}

The majority of patients (88.5\%, Fig. 2) were prescribed an antibiotic after the receipt of their susceptibility testing results. Prescribers were physicians from multiple departments and services, including some services with resident physicians. There was a significant decrease in the average cost of antibiotics per patient after the intervention (1.9 \$ vs. $1.7 \$$, where $1.5 \$$ would mean that the average number of dollar signs for 


\begin{tabular}{|c|c|c|}
\hline & Bacteremia & UTI \\
\hline \multirow[t]{2}{*}{$\begin{array}{l}\text { All admissions with a qualifying ICD- } 9 \text { code } \\
\text { and prescription for antibiotics during } \\
\text { hospital stay }\end{array}$} & 1,688 & 4,577 \\
\hline & 1 & \\
\hline \multirow[t]{2}{*}{$\begin{array}{l}\text { Excluding multiple admissions per person } \\
\text { during the study period }\end{array}$} & 1,471 & 3,902 \\
\hline & $\downarrow$ & \\
\hline \multirow[t]{2}{*}{$\begin{array}{l}\text { Individuals with susceptibility testing during } \\
\text { hospitalization }\end{array}$} & 895 & 226 \\
\hline & $\downarrow$ & \\
\hline \multirow[t]{2}{*}{$\begin{array}{l}\text { Susceptibility testing was on blood } \\
\text { (bacteremia patients) or urine (UTI } \\
\text { patients) sample }\end{array}$} & 648 & 184 \\
\hline & $\psi$ & \\
\hline \multirow[t]{2}{*}{$\begin{array}{l}\text { Susceptibility testing indicated susceptibility } \\
\text { to antibiotics of at least one cost category }\end{array}$} & 648 & 184 \\
\hline & $\downarrow$ & \\
\hline \multirow[t]{2}{*}{$\begin{array}{l}\text { Prescribed antibiotics after susceptibility } \\
\text { testing }\end{array}$} & 581 & 155 \\
\hline & $\downarrow$ & \\
\hline $\begin{array}{l}\text { Final combined group including only the } \\
\text { second susceptibility test result if testing } \\
\text { performed for bacteremia and UTI }\end{array}$ & 652 (564 be & a, $88 \cup \mathrm{UTI})$ \\
\hline
\end{tabular}

Fig. 2 Flow chart of study inclusion criteria for patients with bacteremia or urinary tract infection (UTI)

antibiotics prescribed was between $\$$ and $\$$, pooled $t$-test $\mathrm{t}=$ $-3.1, p=0.002$; Table 1). The change in the frequency of antibiotic prescribing from each cost category was significant $\left(\chi^{2}=13.7, \mathrm{DF}=3, p=0.003\right.$; Fig. 3$)$ and showed a shift toward $\$$ antibiotic prescribing from all higher cost groups. There was no change after the intervention in the proportion of patients prescribed antibiotics to which their infection was resistant (3.0\% before intervention, $3.0 \%$ after intervention; $p=0.945$ ).

\section{Multivariate Logistic Regression}

In our initial model, we included intervention period (pre- or post-cost feedback implementation) and eight other possible exposures influencing prescribing: sex, race, age, insurance status, length of hospital stay prior to antibiotic prescribing, creatinine, SOI, and ROM. This model served as our standard, provided an initial estimate of the odds of prescribing highcost antibiotics $(\$ \$ \$$ or $\$ \$ \$$ ) after the intervention compared

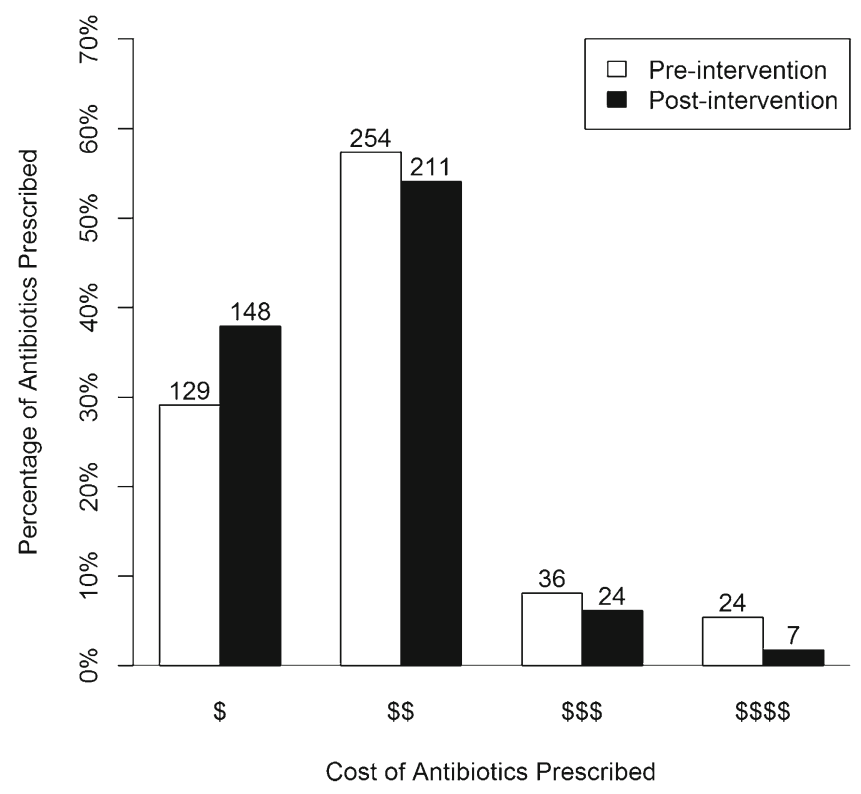

Fig. 3 Histogram of antibiotics prescribed by cost category (\$-\$\$\$) pre- and post-intervention, columns labeled with number of prescriptions. 
to before $(\mathrm{OR}=0.767,95 \% \mathrm{CI}: 0.579,1.016)$, and had no evidence of poor fit (Hosmer-Lemeshow $p=0.686$, cstatistic $=0.670$ ). We then ran models with combinations of the exposure variables to identify models with increased precision but less than $10 \%$ change in the estimated OR. Our final estimated OR for the odds of prescribing high-cost antibiotics after the intervention compared to before was 0.741 (95\% CI: 0.564, 0.976), controlling for the effects of age, length of stay prior to antibiotic prescribing, and insurance type, indicating that the intervention resulted in a statistically significant decrease ( $p=0.033$; Table 2$)$ in the odds of prescribing high-cost antibiotics for inpatients diagnosed with bacteremia or UTI.

\section{Sensitivity Analysis}

Based on our sensitivity analyses, our estimates for the association between the intervention and high-cost antibiotic prescribing were robust (Supplemental Table 2). The OR for the association between cost-feedback and the highest cost antibiotic prescribed per person was 0.772 $(p=0.039)$, controlling for age, length of stay prior to antibiotic prescribing, and insurance type. The OR for the association between cost-feedback and the least expensive antibiotic prescribed per person was $0.879(p=0.078)$, controlling for age, length of stay prior to antibiotic prescribing, and insurance type.

When we stratified by diagnosis, the association between the intervention and antibiotic prescribing remained constant, indicating that the association was consistent for both diagnoses. The OR for the prescription of high-cost antibiotics in the intervention period compared to the pre-intervention period for individuals diagnosed with bacteremia was 0.765 ( $p=0.078$ ), controlling for age, length of stay prior to antibiotic prescribing, and insurance type. The OR for the intervention for individuals diagnosed with UTI was $0.747(p=0.218)$, controlling for age and length of stay prior to antibiotic prescribing. After reassessing both subanalyses using the highest and lowest cost antibiotic prescribed, the ORs remained stable for bacteremia and UTI.

Table 2 Logistic Regression Model Results: Parameters, Odds Ratios, and $95 \%$ Confidence Intervals

\begin{tabular}{llcl}
\hline \hline Variable & $\beta^{*}(\mathbf{S E})$ & $\boldsymbol{p}$-value & OR (95 \% CI) \\
\hline $\begin{array}{l}\text { Intervention (ref. } \\
\text { pre-intervention) }\end{array}$ & $\begin{array}{l}-0.299 \\
(0.140)\end{array}$ & 0.033 & $0.741(0.564,0.976)$ \\
Age & $\begin{array}{l}0.016 \\
(0.009)\end{array}$ & 0.084 & $1.016(0.998,1.034)$ \\
$\begin{array}{l}\text { Length of stay prior } \\
\text { to antibiotic }\end{array}$ & $\begin{array}{l}0.019 \\
\text { prescription }\end{array}$ & $0.006)$ & $1.020(1.006,1.034)$ \\
$\begin{array}{l}\text { Insurance type } \\
\text { (public, ref. other) }\end{array}$ & $\begin{array}{l}-0.234 \\
(0.510)\end{array}$ & 0.646 & $0.791(0.291,2.149)$ \\
$\begin{array}{l}\text { Insurance type } \\
\text { (private, ref. other) }\end{array}$ & $\begin{array}{l}-0.025 \\
(0.296)\end{array}$ & 0.934 & $0.976(0.546,1.743)$ \\
\hline
\end{tabular}

SE standard error, OR odds ratio, CI confidence interval, $D F$ degrees of freedom, ref. reference group ${ }^{* \text { Parameter estimate }}$

\section{Cost-Savings Estimates}

Based on the number of antibiotics prescribed from each category before and after the intervention and the median cost per unit for antibiotics in each cost category, the estimated average cost per unit of antibiotics prescribed before the intervention was $\$ 11.13(\mathrm{SD}=\$ 0.88)$. The estimated average cost per unit of antibiotics prescribed after the intervention was $\$ 7.65(\mathrm{SD}=\$ 0.57)$. This savings of $31.3 \%$ was significant $(p<0.0001)$. There was also a significant difference in the estimated unit cost per patient before and after the intervention ( $\$ 15.86$ per patient vs. $\$ 8.75, p<0.0001$ ). For the 311 patients included in the post-intervention period, this amounts to an estimated $\$ 2,211.21$ or $\$ 7.11$ per patient.

\section{DISCUSSION}

In this quasi-experimental study, adding cost data to culture and antibiotic susceptibility results for inpatients diagnosed with bacteremia or UTI was associated with a significant decrease in the prescription of high-cost antibiotics. We observed reductions in the percentage of antibiotics prescribed from all cost categories greater than $\$$, with the greatest proportional reductions in the highest cost categories, resulting in a significant decrease of approximately $30 \%$ in the average estimated per unit cost of antibiotics prescribed. Assuming a similar savings could be expected to continue beyond the first year following the intervention and may apply to individuals with diagnoses aside from bacteremia or UTI, the potential financial impact may be substantial, especially considering that patients receive multiple antibiotic units per prescription. Our study suggests that providers should incorporate cost data into their prescribing practices.

Our study joins a growing body of literature demonstrating the utility of making providers aware of drug cost at the time of ordering. Although many factors besides cost influence physician prescribing habits, ${ }^{19}$ research demonstrates that many physicians are unaware of drug pricing - overestimating inexpensive drugs and underestimating expensive ones. ${ }^{20}$ However, physicians are receptive to using cost data to guide their prescribing, particularly to minimize patients' out-of-pocket costs. ${ }^{21}$ An important difference between our studies and previous ones is that we used cost categories rather than actual cost.

Two differences between our study and other studies of clinical decision support tools for inpatient prescribing were our intervention's goal and its simplicity. Many tools have been developed to reduce inappropriate antibiotic prescribing. In general, they have been successful at reducing the incidence of negative side effects of excessive or suboptimal antibiotic prescribing, such as $C$. difficile infection, colonization with antibiotic-resistant organisms, and mortality. ${ }^{22}$ The goal of our intervention, however, was purely to reduce cost without negatively impacting patient care. Second, clinical decision support tools can be complex, incorporating 
new screens or requiring click-through to access all information. ${ }^{14,23}$ Our intervention was seamlessly integrated into the existing EHR and did not change the general appearance of the antibiotic susceptibility results screen with which providers were familiar. Despite its simplicity, the intervention was associated with decreases in antibiotic costs, supporting existing literature on the power of "nudges" in improving medical care. ${ }^{24}$

Our study has several strengths. First, we were able to implement our system with minimal information technology (IT) support in a commercially available HER, and it is easily exported to other EHR systems. Second, we coupled cost data with susceptibility testing to ensure that patients received care that was equivalent, from a susceptibility standpoint, but less expensive. The effect of extending the intervention to antibiotics prescribed without sensitivity testing is unknown and might face a variety of challenges. For example, in the absence of sensitivity data, cost categories would have to be integrated into other portions of the EHR and prescribing system. Third, the intervention was both simple and scalable - it did not increase the number of steps needed to order a prescription, did not require any educational programs, and did not involve any incentives. Lastly, although we were unable to do a comprehensive analysis of patient outcomes before and after the intervention, the intervention was not associated with any increase in the average duration of hospital stay or in prescribing of medications to which the cultured pathogen was resistant, suggesting that the antibiotics selected under the cost feedback system may have been similarly effective to those selected before the intervention.

This study also has several limitations. First, the study was a pre-post quasi-experimental analysis; thus, it lacked a control group and was sensitive to unmeasured bias. However, the association was significant even upon sensitivity testing and stratification, which indicates that the findings are robust. Second, the follow-up period lasted 1 year. Its durability was not tested, and we may have instead observed regression to the mean or the Hawthorne effect. Third, the practice setting (an academic teaching institution and tertiary care hospital) may have impacted the intervention. Providers in this setting may have different responses to cost data than those in other practice settings. Fourth, although no antibiotic shortages were noted during the study period and no other cost-related interventions were implemented simultaneously, it is possible that provider habits changed as a result of other social, cultural, or technical factors. Unfortunately, we did not have information on resident physician instruction regarding proper prescribing, changes in the antibiotic stewardship program, or other factors that may have impacted prescribers. Fifth, we did not evaluate the total savings of the intervention across all diagnoses. This remains a topic for future research. Lastly, the population seen in this study had a relatively long length of stay in the hospital and high severity of illness. The effect of the intervention on a less severely ill patient population should be considered in future research.
The intervention did not address the possibility of using cost feedback to guide empiric treatment choices. It is possible that a similar simple feedback mechanism could help guide providers toward selecting appropriate generic or other lower cost treatment options while waiting for culture results. Indeed, other researchers have found that clinical decision support tools that guide physicians to appropriately prescribe antibiotics can have cost benefits and improve patient outcomes. ${ }^{14,22}$ Additional future considerations include the impact of cost feedback systems on readmission rates, clinical failures, and antibiotic resistance patterns.

\section{CONCLUSIONS}

Providing cost data alongside antibiotic susceptibility data for blood and urine culture results may change physicians' ordering behavior. The intervention was low cost, effective, and easy to implement. Whether broadening the intervention by including cost information for all antibiotic prescribing would be effective is a topic worthy of further study, so long as adaptations are made to ensure that patients receive similarly effective prescriptions.

Acknowledgments: The content is solely the responsibility of the authors and does not necessarily represent the official views of the National Institutes of Health.

Funding: This work was supported by funding from the ARCS Foundation and by the National Institute of Diabetes and Digestive and Kidney Diseases of the National Institutes of Health [F30DK100097] to KLN.

Conflict of Interest: The authors declare that they do not have a conflict of interest.

Corresponding Author: Kira L. Newman, B.A.; Department of Epidemiology, Emory University Rollins School of Public Health, 1518 Clifton Rd. NE, Atlanta, GA 30322, USA (e-mail: kira.newman@emory.edu).

\section{REFERENCES}

1. Berwick DM, Hackbarth AD. Eliminating waste in US health care. JAMA. 2012;307:1513-6.

2. Health Information Technology for Economic and Clinical Health (HITECH) Act, Title XIII of Division A and Title IV of Division B of the American Recovery and Reinvestment Act of 2009 (ARRA).

3. Blumenthal D. Stimulating the adoption of health information technology. N Engl J Med. 2009;360:1477-9.

4. Hillestad R, Bigelow J, Bower A, Girosi F, Meili R, Scoville R, et al. Can electronic medical record systems transform health care? Potential health benefits, savings, and costs. Health Aff. 2005;24:1103-17.

5. Chaudhry B, Wang J, Wu S, Maglione M, Mojica W, Roth E, et al. Systematic review: impact of health information technology on quality, efficiency, and costs of medical care. Ann Intern Med. 2006;144:742-52.

6. Evans RS, Classen DC, Pestotnik SL, Lundsgaarde HP, Burke JP. Improving empiric antibiotic selection using computer decision support. Arch Intern Med. 1994;154:878-84.

7. Kaushal R, Shojania KG, Bates DW. Effects of computerized physician order entry and clinical decision support systems on medication safety: a systematic review. Arch Intern Med. 2003;163:1409-16.

8. McCormick D, Bor DH, Woolhandler S, Himmelstein DU. Giving officebased physicians electronic access to patients' prior imaging and lab results did not deter ordering of tests. Health Aff. 2012;31:488-96. 
9. Sidorov J. It ain't necessarily so: the electronic health record and the unlikely prospect of reducing health care costs. Health Aff. 2006;25:107985

10. DesRoches CM, Campbell EG, Vogeli C, Zheng J, Rao SR, Shields AE, et al. Electronic health records' limited successes suggest more targeted uses. Health Aff. 2010;29:639-46.

11. Tierney WM, Miller ME, McDonald CJ. The effect on test ordering of informing physicians of the charges for outpatient diagnostic tests. N Engl J Med. 1990;322:1499-504.

12. Feldman LS, Shihab HM, Thiemann D, Yeh HC, Ardolino M, Mandell S, et al. Impact of providing fee data on laboratory test ordering: a controlled clinical trial. JAMA Intern Med. 2013;173:903-8.

13. Ornstein SM, MacFarlane LL, Jenkins RG, Pan Q, Wager KA. Medication cost information in a computer-based patient record system. Impact on prescribing in a family medicine clinical practice. Arch Fam Med. 1999;8:118-21.

14. McGregor JC, Weekes E, Forrest GN, Standiford HC, Perencevich EN, Furuno JP, et al. Impact of a computerized clinical decision support system on reducing inappropriate antimicrobial use: a randomized controlled trial. J Am Med Inform Assoc. 2006;13:378-84.

15. Fischer MA, Vogeli C, Stedman M, Ferris T, Brookhart MA, Weissman JS. Effect of electronic prescribing with formulary decision support on medication use and cost. Arch Intern Med. 2008;168:2433-9.

16. Schwarz N, Bless H, Bohner G, Harlacher U, Kellenbenz M. Response scales as frames of reference-the impact of frequency-range on diagnostic judgments. Appl Cogn Psychol. 1991;5:37-49.
17. Meurer SJ. Mortality Risk Adjustment Methodology for University Health System's Clinical Data Base. In: Mortality Measurement. Agency for Healthcare Research and Quality, Rockville, MD. November 2008. http:// archive.ahrq.gov/professionals/quality-patient-safety/quality-resources / tools/mortality/Meurer.html. Accessed February 10, 2015.

18. Kleinbaum DG, Klein MK. Logistic Regression: A Self-Learning Text, 3rd Edition. Springer, New York, NY; 2010.

19. Schumock GT, Walton SM, Park HY, Nutescu EA, Blackburn JC, Finley JM, et al. Factors that influence prescribing decisions. Ann Pharmacother. 2004;38:557-62.

20. Allan GM, Lexchin J, Wiebe N. Physician awareness of drug cost: a systematic review. PLoS Med. 2007;4:e283.

21. Khan S, Sylvester R, Scott D, Pitts B. Physicians' opinions about responsibility for patient out-of-pocket costs and formulary prescribing in two Midwestern states. J Manag Care Pharm. 2008; 14:780-9

22. Davey P, Brown E, Charani E, Fenelon L, Gould IM, Holmes A, et al. Interventions to Improve Antibiotic Prescribing Practices for Hospital Inpatients. Cochrane Database of Syst Rev. 2013;4:CD003543.

23. Paul M, Andreassen S, Tacconelli E, Nielsen AD, Almanasreh N, Frank U, et al. Improving empirical antibiotic treatment using TREAT, a computerized decision support system: cluster randomized trial. J Antimicrob Chemother. 2006;58:1238-45.

24. Meeker D, Knight TK, Friedberg MW, Linder JA, Goldstein NJ, Fox CR, et al. Nudging guideline-concordant antibiotic prescribing: a randomized clinical trial. JAMA Intern Med. 2014;174:425-31. 